\title{
A novel homozygous missense SLC25A20 mutation in three CACT-deficient patients: clinical and autopsy data
}

\author{
Yasutsugu Chinen ${ }^{1,2}$, Kumiko Yanagi ${ }^{3}$, Sadao Nakamura ${ }^{1}$, Noriko Nakayama', Motoko Kamiya ${ }^{4,7}$, Mami Nakayashiro ${ }^{5}$,
} Tadashi Kaname $\mathbb{B}^{3}$, Kenji Naritomi ${ }^{6}$ and Koichi Nakanishi $\mathbb{B}^{1,2}$

\begin{abstract}
Carnitine-acylcarnitine translocase (CACT) deficiency is a fatty acid B-oxidation disorder of the carnitine shuttle in mitochondria, with a high mortality rate in childhood. We evaluated three patients, including two siblings, with neonatal-onset CACT deficiency and revealed identical homozygous missense mutations of p.Arg275Gln within the SLC25A20 gene. One patient died from hypoglycemia and arrhythmia at 26 months; his pathological autopsy revealed increased and enlarged mitochondria in the heart but not in the liver.
\end{abstract}

Mitochondrial fatty acid ß-oxidation has a pivotal role in energy production during starvation, particularly in the heart and skeletal muscle, because they depend mainly on the oxidation of long-chain fatty acids. The metabolism of cytosolic long-chain fatty acids into acyl-CoA occurs in the intramitochondrial space via the carnitine shuttle, which consists of carnitine palmitoyltransferase (CPT) I, CPT II, and carnitine-acylcarnitine translocase $(\mathrm{CACT})^{1-3}$. High levels of CACT mRNA transcripts, encoded by the SLC25A20 gene on chromosome 3p21 (MIM*613698), are present in the heart, skeletal muscle, and liver; lower levels are present in the brain, kidney, pancreas, and lung $^{4,5}$. CACT deficiency (MIM212138) was first described in $1992^{6}$; it is a rare autosomal-recessive disease of mitochondrial fatty acid oxidation with a high mortality rate (65\%). In CACT deficiency, abnormal acylcarnitine profiles revealed high levels of C16-carnitine and (C16+ C18:1)/C2, including C14/C3, similar to those found in CPT II deficiency in a mass screening of newborns ${ }^{7}$. Most

\footnotetext{
Correspondence: Yasutsugu Chinen (ychinen@med.u-ryukyu.ac.jp)

${ }^{1}$ Department of Child Health and Welfare (Pediatrics), Graduate School of Medicine, University of the Ryukyus, Nishihara, Okinawa, Japan

${ }^{2}$ Genetic Counseling Room, University of the Ryukyus Hospital, Nishihara, Okinawa, Japan

Full list of author information is available at the end of the article
}

patients (82\%) with neonatal-onset CACT deficiency have hypoketotic hypoglycemia, hyperammonemia, skeletal muscle weakness, and cardiomyopathy with arrhythmia, leading to cardiac arrest ${ }^{8}$. To prevent adipose tissue lipolysis, sufficient intake of glucose to maintain normal plasma glucose levels, the intake of medium-chain triglycerides (MCTs), and a restriction of long-chain fatty acid intake is required. Brivet (2004) recommended that L-carnitine therapy be administered intravenously, with intravenous injection of high concentrations of glucose in emergencies and orally 3-4 times/day when the patient is stable $^{9}$. Despite oral carnitine supplementation, plasma carnitine levels remain very low in CACT-deficient patients.

At the Pediatric Department of the University of the Ryukyus, three patients in two unrelated families (Fig. 1: Family-a, -b) from Okinawa, Japan, with neonatal onset of CACT deficiency diagnosed by metabolite and genomic mutation analyses, were followed up from February 2008 to October 2017. After direct Sanger sequencing for all exons of $S L C 25 A 20$, a homozygous missense variant of NM_000387.6:c.824G>A (p.Arg275Gln) in the SLC25A20 gene (Fig. 2a) was identified in the three patients [P1, P2, P3], included two siblings [P2, P3]. The affected amino acid is evolutionarily conserved from fungi to humans, 


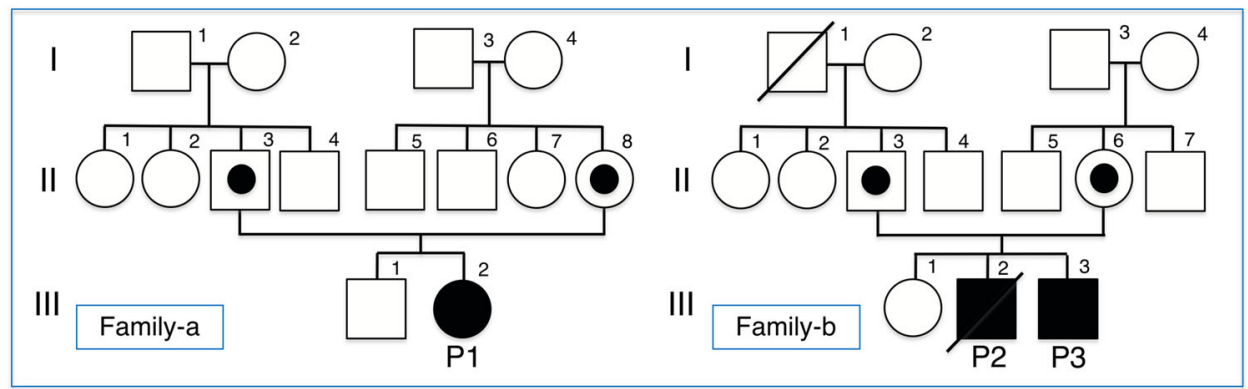

Fig. 1 Two family pedigrees with results of the analysis of the SLC25A20 gene. To our knowledge, there is no apparent consanguinity between the two families. p.Arg275GIn: Family-a; II-3 and II-8 (heterozygosity), III-2 (homozygosity), Family-b; II-3 and II-6 (heterozygosity), III-2 and III-3 (homozygosity)
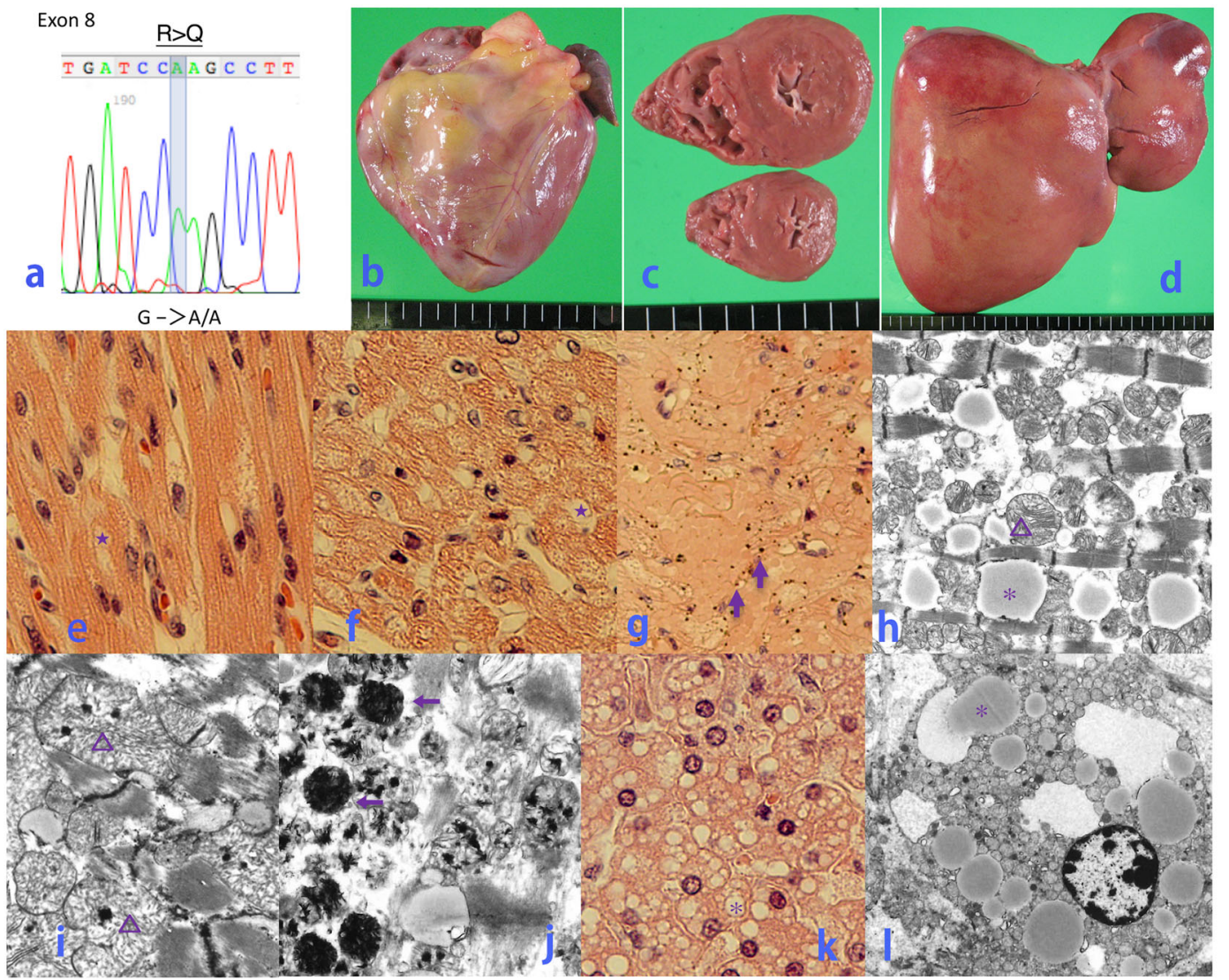

Fig. 2 Postmortem pathological imaging and the analysis of the SLC25A20 gene of P2 at $\mathbf{2 6}$ months of age. a Molecular analysis of the SLC25A20 gene revealed homozygous missense mutations of p.Arg275Gln. b, c, e-j Heart-mild myocardial thickening, weight $70 \mathrm{~g}$ (+0.5 SD). b frontal view; c transverse ventricle; e hematoxylin-eosin (HE) stain $\times 400$; $\mathbf{f} H E$ stain $\times 100$, partial vacuolation (star), hypertrophy, and eosinophilic changes in cardiomyocytes; $\mathbf{g}$ HE stain $\times 200$, the deposition of small dark-brown granules ( $\uparrow$ ); $\mathbf{h}$-j electron microscopic imaging (EMI) showing small lipid droplets $(*)$, increased numbers of mitochondria, the appearance of giant mitochondria (open triangle), and the deposition of amorphous materials with high electron density $(\leftarrow)$ in mitochondria. $\mathbf{h} \times 2300 ; \mathbf{i} \times 6900 ; \mathbf{j} \times 11,500$. d, $\mathbf{k}$, I Liver-hepatic steatosis, weight $360 \mathrm{~g}(-1.0 \mathrm{SD})$. d light yellow liver, frontal view; $\mathbf{k}$ HE stain $\times 200$, diffuse deposition of small- to medium-sized lipid droplets $\left(^{*}\right)$ in liver cells; I EMI $\times 2300$, the deposition of small lipid droplets $\left(^{*}\right)$ in all hepatocytes, no changes in the liver mitochondria. Scale $=0.5 \mathrm{~cm}(\mathbf{a}, \mathbf{b}, \mathbf{d})$.

emphasizing that the residue is likely to be functionally important. This was predicted to be a disease-causing mutation by PolyPhen-2 software (http://genetics.bwh. harvard.edu/pph/) and Mutation Taster (http://www. mutationtaster.org), with normal activity of the CPT II enzyme retained $[\mathbf{P 1}]$. Although we could not find this 
variant in ClinVar (https://www.ncbi.nlm.nih.gov/clinvar/) and the Japanese genome database of Human Genetic Variation (http://www.hgvd.genome.med.kyoto-u.ac.jp), we found it in gnomAD (https://gnomad.broadinstitute. org). The frequency of this variant is very low: 0.00002784 [allele count: 7/251396, east Asian: 1/18394, European (non-Finnish): 6/113684)] and has no number of homozygotes. Biochemical analysis of P1, P2, and P3 demonstrated high levels of C16 acylcarnitine (14.55, 2.61, 8.61; cut-off $>2.1[\mathrm{nmol} / \mathrm{ml}]$ ) and high ratios of C14 to C3 $(6.06,1.03,1.55$; cut-off $>0.408)$ and $(\mathrm{C} 16+\mathrm{C} 18: 1)$ to $\mathrm{C} 2$ (3.70, 1.83, 0.79; cut-off $>0.41)$, as measured in dried blood spots by tandem mass spectrometry.

P1 was born at 34 weeks and 6 days of gestation with a birth weight of $1844 \mathrm{~g}(-1.3 \mathrm{SD})$. She was transferred to a neonatal intensive care unit for observation. At 2 days of age, she suffered from tachypnea, bradycardia, and hyperammonemia $(>1000 \mu \mathrm{g} / \mathrm{dl})$ [normal range: $36-86 \mu \mathrm{g} / \mathrm{dl}]$. She was treated with continuous arteriovenous hemodialysis and L-carnitine and recovered, but she experienced intracranial bleeding. She then received L-carnitine supplementation and was fed a fat-restricted diet. She was hospitalized many times because of infection. At 5 years of age, magnetic resonance imaging (MRI) of her brain demonstrated no abnormal findings, and an echocardiogram revealed no hypertrophic cardiomyopathy. Developmental assessment by the Kyodai-shiki schedule (Kyoto International Social Welfare Exchange Center) identified mild mental retardation (developmental quotient $[D Q]=75)$ at 4 years and 7 months of age; at 10 years and 8 months, her DQ was 63, her height was $137.4 \mathrm{~cm} \quad(-0.6 \mathrm{SD})$, and her weight was $30.8 \mathrm{~kg}$ $(-0.7 \mathrm{SD})$.

P2 was born at 39 weeks of gestation with a birth weight of $2570 \mathrm{~g}(-1.0 \mathrm{SD})$. At 2 days of age, he presented with grunting, hypoxemia, hypoglycemia $(<40 \mathrm{mg} / \mathrm{dl})$, arrhythmia (wide QRS, ventricular tachycardia), and hyperammonemia $(300-400 \mu \mathrm{g} / \mathrm{dl})$. He recovered after the administration of high concentrations of glucose and an intravenous L-carnitine infusion. At 5 days of age, aspirin was administered into the portal vein for thrombosis. This was followed by L-carnitine supplementation, MCT milk, and a fat-restricted diet. At 26 months of age, after playing on the beach, he presented with a tonicclonic seizure for $20 \mathrm{~min}$, hypoglycemia, and arrhythmia (wide QRS). He was transferred to our hospital but died of cardiac arrest despite the administration of $20 \%$ glucose infusion, epinephrine, and L-carnitine. Postmortem examination showed mild myocardial thickening and hepatic steatosis (Fig. 2b-d, k). His heart weight was $70 \mathrm{~g}$ $(+0.5 \mathrm{SD})[64.9 \pm 10.05 \mathrm{~g}$ at 2 years of age], liver weight was $360 \mathrm{~g}(-1.0 \mathrm{SD})$ [ $463.81 \pm 103.51 \mathrm{~g}$ at 2 years of age], and the width of the left ventricle wall was $0.97 \mathrm{~mm}$ $(+1.8 \mathrm{SD})[0.70 \pm 0.15 \mathrm{~mm} \text { at } 2 \text { years of age }]^{10}$. The postmortem pathological findings of P2 showed partial vacuolation, hypertrophy, and eosinophilic changes in cardiomyocytes (Fig. 2e-f), with small dark-brown granules being found in some areas (Fig. 2g). No invasion of inflammatory cells or myocardial necrosis was observed. Electron microscopic imaging (EMI) of cardiomyocytes showed the presence of small lipid droplets, increased numbers of mitochondria, the appearance of giant mitochondria, and the deposition of amorphous materials with high electron density in mitochondria (Fig. $2 \mathrm{~h}-\mathrm{j}$ ). The diffuse deposition of small- to medium-sized lipid droplets in liver cells was notable (Fig. 2k), with small lipid droplets being observed in all hepatocytes (Fig. 2l). Ambiguous vacuoles were also frequently present in hepatocytes. In contrast to the myocardial mitochondria (Fig. $2 \mathrm{~h}-\mathrm{j}$ ), there were no changes observed in liver mitochondria (Fig. 2l).

P3, a younger brother of P2, was born at 38 weeks of gestation with a birth weight of $2676 \mathrm{~g}(-0.7 \mathrm{SD})$, length of $47.8 \mathrm{~cm}(-0.5 \mathrm{SD})$, and head circumference of $32 \mathrm{~cm}$ $(-0.7 \mathrm{SD})$. He had good activity at birth; however, $30 \mathrm{~min}$ after birth, he had hypoglycemia $(24 \mathrm{mg} / \mathrm{dl})$ [normal range: $50-120]$, and he was treated with $10 \%$ glucose. Five hours after birth, he had apnea and was treated with an intravenous infusion of L-carnitine $(100 \mathrm{mg} / \mathrm{kg} /$ day $)$ and MCT milk (formula 721, Meiji Dairies Corporation, Tokyo, Japan). At 7 months of age, he failed to thrive and received tube feeding. At 11 months of age, an echocardiogram revealed hypertrophic cardiomyopathy. His left ventricular ejection fraction (LVEF) was 35\% (normal range $>55 \%$ ), and his level of N-terminal pro-B-type natriuretic peptide (NT-proBNP, a cardiac biomarker of heart failure and cardiomyopathy ${ }^{11,12}$ ) was $2176 \mathrm{pg} / \mathrm{ml}$ (normal range: $0.0-54.9$ ). In the hospital, an intravenous infusion of L-carnitine $(100 \mathrm{mg} / \mathrm{kg} /$ day $)$ was continuously administered for 14 days. Subsequently, his level of NTproBNP decreased from 2897 to $599.4 \mathrm{pg} / \mathrm{ml}$, and LVEF increased to $44.8 \%$. At 12 months of age, P3 could walk alone; at 13 months of age, he had a gastrostomy for tube feeding. At 4 years and 1 month of age, an echocardiogram and chest X-ray demonstrated dilated cardiomyopathy (LVEF 63\%, left ventricular internal dimension in diastole (LVIDd) $50.5 \mathrm{~mm}$ [z score 3.12], CTR 58\%, trivial mitral regurgitation). At 4 years and 5 months of age, the Kyodai-shiki developmental schedule identified mild mental retardation $(D Q=57)$. At 5 years of age, magnetic resonance imaging of his brain presented no abnormal findings. At 5 years and 6 months of age, his height was $112.4 \mathrm{~cm}(+0.5 \mathrm{SD})$, and his weight was $24.1 \mathrm{~kg}(+1.7 \mathrm{SD})$. He took simple meals orally, in combination with tube feeding.

In P1 and P2, metabolic decompensation with hyperammonemia in the neonatal period was improved by the administration of high concentrations of glucose and 
L-carnitine; in P3, cardiomyopathy was ameliorated by continuous intravenous infusion of L-carnitine in the hospital at 7 months of age. Our report suggests that Lcarnitine might improve cardiac conditions and the general condition under conditions of normal blood glucose levels, as has been previously stated ${ }^{9,13,14}$.

When the hypoglycemia of P2 did not improve, the administration of L-carnitine was ineffective. P2 died of hypoglycemia and arrhythmia, and his autopsy demonstrated that mitochondria in the heart were increased and enlarged, whereas they were unchanged in the liver. When cytochrome c or ATP synthase is insufficient, mitochondria compensate by increasing in size and number ${ }^{15-17}$. Because there were no fatal pathological changes-such as myocardial necrosis-observed in the autopsy of P2, P2 might have had a fatal arrhythmia. Specifically, insufficient ATP production, or mitochondrial dysfunction due to an imbalance in the acyl-CoA:CoA ratio, along with the increased production of long-chain fatty acids caused by hypoglycemia, might have led to changes in the electrical activity of myocardial cells. To our knowledge, this is the first description of autopsy findings of CACT deficiency. As there are currently insufficient clinical and fundamental data regarding the mechanism of lethal arrhythmia in CACT deficiency, further investigations are warranted.

\section{HGV database}

The relevant data from this Data Report are hosted at the Human Genome Variation Database at https://doi.org/10.6084/m9.figshare.hgv.2841.

\section{Acknowledgements}

We thank Ryuji Tasaki of KM Biologics (Kumamoto, Japan) and Yoshino Takayama, Rina Yamashiro, and Yoko Matayoshi of the Medical Examination Center of Chubu Medical Association (Okinawa, Japan) for the analyses by tandem mass spectrometry. We are indebted to the patients and their parents, nurses, and physicians who supported this study. We thank Gemma Richards, PhD, of the Edanz Group (www.edanzediting.com/ac) for editing a draft of this manuscript. This work was supported in part by a grant from the Initiative on Rare and Undiagnosed Diseases in Pediatrics (IRUD-P) project (16ek0109166h0002) from the Japan Agency for Medical Research and Development (AMED).

\section{Author details}

'Department of Child Health and Welfare (Pediatrics), Graduate School of Medicine, University of the Ryukyus, Nishihara, Okinawa, Japan. ${ }^{2}$ Genetic Counseling Room, University of the Ryukyus Hospital, Nishihara, Okinawa, Japan. ${ }^{3}$ Department of Genome Medicine, National Center for Child Health and Development, Tokyo, Japan. ${ }^{4}$ Department of Pediatrics, Naha City Hospital, Naha, Okinawa, Japan. ${ }^{5}$ Department of Pediatrics, Okinawa Prefectural Nanbu Medical Center Children's Medical Center, Haebaru, Okinawa, Japan. ${ }^{6}$ Okinawa Nanbu Habilitation and Medical Center, Naha, Japan. ${ }^{7}$ Present address: Center for Medical Genetics, Shinshu University Hospital, Matsumoto, Japan
Conflict of interest

The authors declare that they have no conflict of interest.

\section{Publisher's note}

Springer Nature remains neutral with regard to jurisdictional claims in published maps and institutional affiliations.

Received: 9 May 2019 Revised: 26 March 2020 Accepted: 26 March 2020. Published online: 16 April 2020

\section{References}

1. Palmieri, F. Diseases caused by defects of mitochondrial carriers: a review. Biochim Biophys. Acta 1777, 564-578 (2008).

2. Houten, S. M. \& Wanders, R. J. A general introduction to the biochemistry of mitochondrial fatty acid-oxidation. J. Inherit. Metab. Dis. 33, 469-477 (2010).

3. Liang, W. C. \& Nishino, I. Lipid storage myopathy. Curr. Neurol. Neurosci. Rep. 11 97-103 (2011).

4. Huizing, M. et al. Cloning of the human carnitine-acylcarnitine carrier CDNA and identification of the molecular defect in a patient. Am. J. Hum. Genet 61, 1239-1245 (1997).

5. Wang, G. L. et al. Expanded molecular features of carnitine acyl-carnitine translocase (CACT) deficiency by comprehensive molecular analysis. Mol. Genet Metab. 103, 349-357 (2011).

6. Stanley, C. A. et al. A deficiency of carnitine-acylcarnitine translocase in the inner mitochondrial membrane. N. Engl. J. Med. 327, 19-23 (1992).

7. Tajima, G. et al. Newborn screening for carnitine palmitoyltransferase II deficiency using (C16 + C18:1)/C2: Evaluation of additional indices for adequate sensitivity and lower false-positivity. Mol. Genet. Metab. 122, 67-75 (2017).

8. Vitoria, I. et al. Carnitine-acylcarnitine translocase deficiency: experience with four cases in Spain and review of the literature. JIMD Rep. 20, 11-20 (2015).

9. Brivet M. Carnitine-acylcarnitine translocase deficiency. Orphanet Encyclopedia. 1-5 (2004).

10. Questionnaire research performed by the Japanese Society of Legal Medicine: weights and sizes of internal organs measured in forensic autopsy cases from 2009 to 2013 in Japan. Planning and Investigation Committee of the Japanese Society of Legal Medicine. www.jslm.jp/problem/zouki.pdf.

11. Magga, J. et al. Significance of plasma levels of N-terminal Pro-B-Type natriuretic peptide on left ventricular remodeling in non-obstructive hypertrophic cardiomyopathy attributable to the Asp175Asn mutation in the atropomyosin gene. Am. J. Cardiol. 101, 1185-1190 (2008).

12. Sherbuk, J. E. et al. BNP, NTproBNP, CMBK, and MMP-2 predict mortality in severe chagas cardiomyopathy. Glob. Hear 10, 173-180 (2015).

13. Roe, C. R. \& Brunengraber, H. Anaplerotic treatment of long-chain fat oxidation disorders with triheptanoin: review of 15 years experience. Mol. Genet. Metab. 116, 260-268 (2015).

14. lacobazzi, V. et al. Response to therapy in carnitine/acylcarnitine translocase (CACT) deficiency due to a novel missense mutation. Am. J. Med. Genet. A 126, 150-155 (2004).

15. Hoppel, C. L., Tandler, B., Fujioka, H. \& Riva, A. Dynamic organization of mitochondria in human heart and in myocardial disease. Int J. Biochem. Cell Biol. 41, 1949-1956 (2009).

16. Vickers, A. E., Bentley, P. \& Fisher, R. L. Consequences of mitochondrial injury induced by pharmaceutical fatty acid oxidation inhibitors is characterized in human and rat liver slices. Toxicol. Vitr. 20, 1173-1182 (2006).

17. $\mathrm{Pu}, \mathrm{J}$. et al. Interactomic study on interaction between lipid droplets and mitochondria. Protein Cell 2, 487-496 (2011). 